\title{
Immune checkpoint inhibitors in elderly patients treated for a lung cancer: a narrative review
}

\author{
Charles Naltet ${ }^{1,2}$, Benjamin Besse ${ }^{1,3}$ \\ ${ }^{1}$ Cancer Medicine Department, Gustave Roussy, Villejuif, France; ${ }^{2}$ Department of Pulmonology and Thoracic Oncology, Hôpital Saint Joseph, Paris, \\ France; ${ }^{3}$ Paris-Sacaly University, Orsay, France \\ Contributions: (I) Conception and design: Both authors; (II) Administrative support: Both authors; (III) Provision of study materials or patients: None; \\ (IV) Collection and assembly of data: Both authors; (V) Data analysis and interpretation: Both authors; (VI) Manuscript writing: Both authors; (VII) \\ Final approval of manuscript: Both authors. \\ Correspondence to: Charles Naltet. Cancer Medicine Department, Gustave Roussy, Villejuif, France; Department of Pulmonology and Thoracic \\ Oncology, Hôpital Saint Joseph, Paris, France. Email: Charles.naltet@gustaveroussy.fr.
}

\begin{abstract}
This article is a review of the literature concerning efficacy and safety of immune checkpoint inhibitors (ICIs) in the elderly population. In the past decade, immunotherapy deeply changed the treatment paradigm of lung cancer in particular in advanced non-small cell lung cancer (aNSCLC). Thus, ICIs have successively demonstrated a survival benefit as single agent in second line, and moved in first line as monotherapy for patients with high programmed death protein 1 (PD-L1) expression or in combination with chemotherapy regardless PD-L1 expression. If patients aged 70 years or older represent up to half of our patients in clinical routine, elderly population is significantly under-represented in clinical trials. This leads to a lack of knowledge concerning efficacy and safety of ICIs in a population of patients with frequent comorbidities, organs dysfunctions and a potential immune-senescence due to age. In this review, we described available data evaluating efficacy and safety of ICI either as monotherapy or in combination in elderly population treated for a lung cancer. These data derived from clinical trial evaluating ICIs in aNSCLC as single agent or in combination with chemotherapy or anti-cytotoxic T-lymphocyte antigen 4 (CTLA-4). As monotherapy, older patients seem to derive the same benefit from ICIs than younger patients with no excess of toxicities. In combination with chemotherapy, real impact of ICIs in elderly population is still unclear. Results of dedicated studies evaluating ICIs as single agent or in combination in elderly patients are needed.
\end{abstract}

Keywords: Elderly; lung cancer; non-small cell lung cancer (NSCLC); small cell lung cancer; immune checkpoint inhibitors (ICIs); anti-programmed death protein 1 (anti-PD-1); anti-programmed death ligand 1 (anti-PD-L1); immunotherapy

Submitted Dec 05, 2020. Accepted for publication Jun 08, 2021.

doi: $10.21037 /$ tlcr-20-1239

View this article at: http://dx.doi.org/10.21037/tlcr-20-1239

\section{Introduction}

Lung cancer is the leading cause of cancer-related death worldwide. Most of the time patients are diagnosed with a metastatic disease that explains a poor prognosis. Non-small cell lung cancer (NSCLC), the most common subtype of lung cancer is predominantly diagnosed in elderly patients (1). According to the SEER database, patients aged 70 years or older represented $43 \%$ of newly diagnosed NSCLC of whom $14 \%$ were 80 years or older (2). Despite this, elderly patients are significantly under-represented in clinical trials. Thereby, in a FDA analysis from a 10 -year experience, only $24 \%$ of patients enrolled in clinical trials were 70 years or older (3). In 2013, Sacher et al. highlighted that the median age of patients with NSCLC enrolled in 248 trials between 1980 and 2010 was 60.9 years and $33 \%$ of these trials 
especially excluded elderly population (4). That leads to a lack of knowledge in a population of patients with frequent comorbidities and organs dysfunctions (5). In addition, elderly patients enrolled in clinical trials are often fitter than the population treated in daily practice.

In the past decade, immunotherapy deeply changed the treatment paradigm of lung cancer. Chemotherapy has been associated with a low response rate (RR), decrease quality of life and high risk of treatment-related toxicities (6) whereas targeted therapies were limited to a small subset of molecularly selected patients. Immune checkpoints inhibitors (ICI), in particular Abs that target programmed death protein 1 (PD-1) or programmed death ligand 1 (PDL1) have demonstrated a benefit in term of survivals as monotherapy compared to single agent chemotherapy in pretreated metastatic NSCLC (7-10). More recently, ICI were successfully investigated in frontline as monotherapy in subgroup of advanced NSCLC (aNSCLC) with high PDL1 expression (11) or in combination with chemotherapy in aNSCLC and extensive-stage small cell lung cancer (ESSCLC) independently of PD-L1 expression. Targeting the host's immune system in order to restore anti-tumor activity, ICI may have less benefit in the elderly whose aging is associated with a functional decline of immune system called immune-senescence (12). Unfortunately, specific clinical trials using ICI in older-adults with aNSCLC are still lacking.

In this review, we describe available data evaluating efficacy and safety of ICI either as monotherapy or in combination in elderly population treated for a lung cancer. We present the following article in accordance with the narrative review reporting checklist (available at http:// dx.doi.org/10.21037/tlcr-20-1239).

\section{Methods}

A PubMed search for English-language articles was performed until November 2020. We focused our research on meta-analysis, phase II trials and phase III randomized clinical trials evaluating ICI in aNSCLC or ES-SCLC that have influenced the standard of care (SoC) in these situations as monotherapy or in combination. We also included clinical trials describing real-world analysis of ICI in lung cancer or Immune related adverse event.

\section{Single agent ICI efficacy in aNSCLC}

ICI were first evaluated as monotherapy in second or further line of aNSCLC. The Food and Drug administration (FDA) and the European Medicine Agency (EMA) have approved three drugs (Nivolumab, Atezolizumab and Pembrolizumab) in this situation since 2015. All demonstrated a clear benefit on overall survivals (OSs) compared to SoC (Table 1). These drugs have also been investigated in first line compared to platinum-based regimen with more conflicting results (Figure 1).

\section{Nivolumab}

Nivolumab is a fully human IgG4 PD-1 monoclonal antibody (mAb). In CheckMate 063, a single arm phase II study, 117 patients with squamous aNSCLC previously treated received Nivolumab $3 \mathrm{mg} / \mathrm{kg}$ every 2 weeks. Median age was 65 years and $14 \%$ of patients $(n=16 / 117)$ were at least 75 years. RR was $14.5 \%$ in the overall population, $16 \%$ $(\mathrm{n}=16 / 101)$ in patients $<75$ years compared to $6.2 \%(\mathrm{n}=1 / 16)$ in the elderly population ( $\geq 75$ years) (13).

In CheckMate 057, a phase III trial, 582 previously treated nonsquamous aNSCLC were randomly assigned to Nivolumab $3 \mathrm{mg} / \mathrm{kg}$ every 2 weeks $(\mathrm{n}=292)$ or Docetaxel $75 \mathrm{mg} / \mathrm{m}^{2}$ every 3 weeks $(\mathrm{n}=290)$. The median age was 62 years and 43 patients $(7 \%)$ had at least 75 years. In the overall population, Nivolumab demonstrated a benefit in OS with a median OS (mOS) of 12.2 months compared to 9.4 months in control arm [hazard ratio (HR), 0.73; $96 \% \mathrm{CI}, 0.59$ to 0.89$]$. In the subgroup of patients aged at least 75 years, the benefit was uncertain (HR, 0.90; $95 \%$ CI, 0.43 to 1.87 ) (8). The phase III trial CheckMate 017 evaluated Nivolumab in previously treated patient with squamous aNSCLC using the same design than CheckMate 057. This study enrolled 272 patients, 135 patients randomly assigned in Nivolumab group and 137 patients in Docetaxel group. The median age was 63 years with $11 \%(n=29 / 272)$ patients aged at least 75 years. In the overall population, Nivolumab showed a significant improvement of OS with mOS of 9.2 versus 6.0 months (HR $0.59 ; 95 \% \mathrm{CI}, 0.44$ to 0.79 ), but it seemed to be deleterious for elderly population (HR 1.85 ; $95 \%$ CI, 0.76 to 4.51 ) in the subgroup analysis (7). CheckMate 078 was a multinational phase III trial evaluating Nivolumab as single agent compared to Docetaxel in pre-treated aNSCLC. In this study, the median age was 60 years and only $2 \%(n=12 / 504)$ of patients were 75 or older. Nivolumab demonstrated an OS benefit in the overall population (HR $0.68 ; 95 \%$ CI, 0.52 to 0,90 ) and in the subgroup of patients aged at least 65 years (14). 
Table 1 Phase III studies of anti-PD-1 or PD-L1 mAbs in aNSCLC with subgroup analysis data in the elderly population

\begin{tabular}{|c|c|c|c|c|c|c|c|c|c|c|c|c|}
\hline \multirow{2}{*}{ Author (year) } & \multirow{2}{*}{ Study } & \multirow{2}{*}{ Phase } & \multirow{2}{*}{ Histology } & \multirow{2}{*}{ Line } & \multirow{2}{*}{ Treatment arms } & \multirow{2}{*}{ No. of patients } & \multirow{2}{*}{ Median age (years) } & \multirow{2}{*}{ Age cut-off, older patients } & \multirow{2}{*}{ No. of older [\%] } & \multicolumn{3}{|c|}{ Overall survivals } \\
\hline & & & & & & & & & & Overall HR (95\% Cl) & HR for younger $(95 \% \mathrm{Cl})$ & HR for older $(95 \% \mathrm{Cl})$ \\
\hline Borghaei (2015) & CheckMate 057 & III & Nonsquamous NSCLC & $>1$ & Nivo vs. Docetaxel & 582 & 61.0 [37-84]; 64.0 [21-85] & $65-74 ; \geq 75$ & 200 [34]; 43 [7] & $0.73(0.59-0.89)$ & $0.81(0.62-1.04)$ & $\begin{array}{l}0.63(0.45-0.89) ; \\
0.90(0.43-1.87)\end{array}$ \\
\hline Brahmer (2015) & CheckMate 017 & III & Squamous NSCLC & $>1$ & Nivo vs. Docetaxel & 272 & 62.0 [39-85]; 64 [42-84] & $65-74 ; \geq 75$ & 91 [33]; 29 [11] & $0.59(0.44-0.79)$ & $0.52(0.35-0.75)$ & $\begin{array}{l}0.56(0.34-0.91) ; \\
1.85(0.76-4.51)\end{array}$ \\
\hline Carbone (2017) & CheckMate 026 & III & NSCLC & 1 & Nivo vs. PBCh & 423 & 63 [32-89]; 65 [29-87] & $\geq 65: 65-74 ; \geq 75$ & 260 [48]: 198 [37]; 62 [11] & $1.08(0.87-1.34)$ & $1.13(0.83-1.54)$ & $1.04(0.77-1.41)$ \\
\hline Herbst (2016) & Keynote-010 & $\| / I I I$ & NSCLC & $>1$ & $\begin{array}{l}\text { Pembro } 2 \mathrm{mg} / \mathrm{kg} \text { vs. Pembro } \\
10 \mathrm{mg} / \mathrm{kg} \text { vs. Docetaxel }\end{array}$ & 1,034 & $\begin{array}{c}63 \text { [56-69]; 63 [56-69]; } \\
62 \text { [56-69] }\end{array}$ & $\geq 65$ & $429[41]$ & $\begin{array}{l}0.71(0.58-0.88) ; \\
0.61(0.49-0.75)\end{array}$ & $\begin{array}{l}0.63(0.50-0.79) ; \\
\text { pooled-analysis }\end{array}$ & $\begin{array}{l}0.76(0.57-1.02) ; \\
\text { pooled-analysis }\end{array}$ \\
\hline Reck (2019) & Keynote-024 & III & NSCLC & 1 & Pembro vs. PBCh & 305 & 64.5 [33-90]; 66.0 [28-85] & $\geq 65$ & 164 [54] & $0.63(0.47-0.86)$ & $0.60(0.38-0.96)$ & $0.64(0.42-0.98)$ \\
\hline Fehrenbacher (2018) & OAK & III & NSCLC & $>1$ & Atezo vs. Docetaxel & 1,225 & 63.0 [25-84]; 64.0 [34-85] & $\geq 65$ & $564[46]$ & $0.80(0.70-0.92)$ & $0.84(0.70-1.01)$ & $0.75(0.61-0.91)$ \\
\hline Spigel (2019) & IMpower-110 & III & NSCLC & 1 & Atezo vs. PBCh & 554 & $\mathrm{NA} ; \mathrm{NA}$ & TC3/IC3, $\geq 65: 65-74 ; \geq 75$ & 103 [50]: 80 [39]; 22 [11] & TC3/C3: $0.59(0.40-0.89)$ & TC3/IC3: 0.59 (0.34-1.04) & $\begin{array}{c}\text { TC3/IC3: } 0.63(0.34-1.19) ; \\
1.04(0.19-5.70)\end{array}$ \\
\hline Planchard (2020) & ARCTIC Study A & . 111 & NSCLC & $>2$ & Durva vs. SoC & 126 & $63.5[35-79] ; 62.0[41-81]$ & $\geq 65$ & $56[44]$ & $0.63(0.42-0.93)$ & NA & NA \\
\hline $\operatorname{Rizvi}(2020)$ & MYSTIC & IIII & NSCLC & 1 & $\begin{array}{c}\text { Durva vs. Durva + Treme vs. } \\
\text { PBCh }\end{array}$ & TPS $\geq 25: 489$ & $\begin{array}{c}64.0 \text { [32-84]; } 65.0 \text { [64-87]; } \\
64.5[35-85]\end{array}$ & $\geq 65$ & 256 [52] & $\begin{array}{c}0.76(0.56-1.02) ; 0.85(0.61- \\
1.17)\end{array}$ & $0.86(0.60-1.24) ; \mathrm{NA}$ & $0.66(0.45-0.95) ; \mathrm{NA}$ \\
\hline
\end{tabular}

Tremelimumab; NA, not available; TPS, tumor proportion score. 


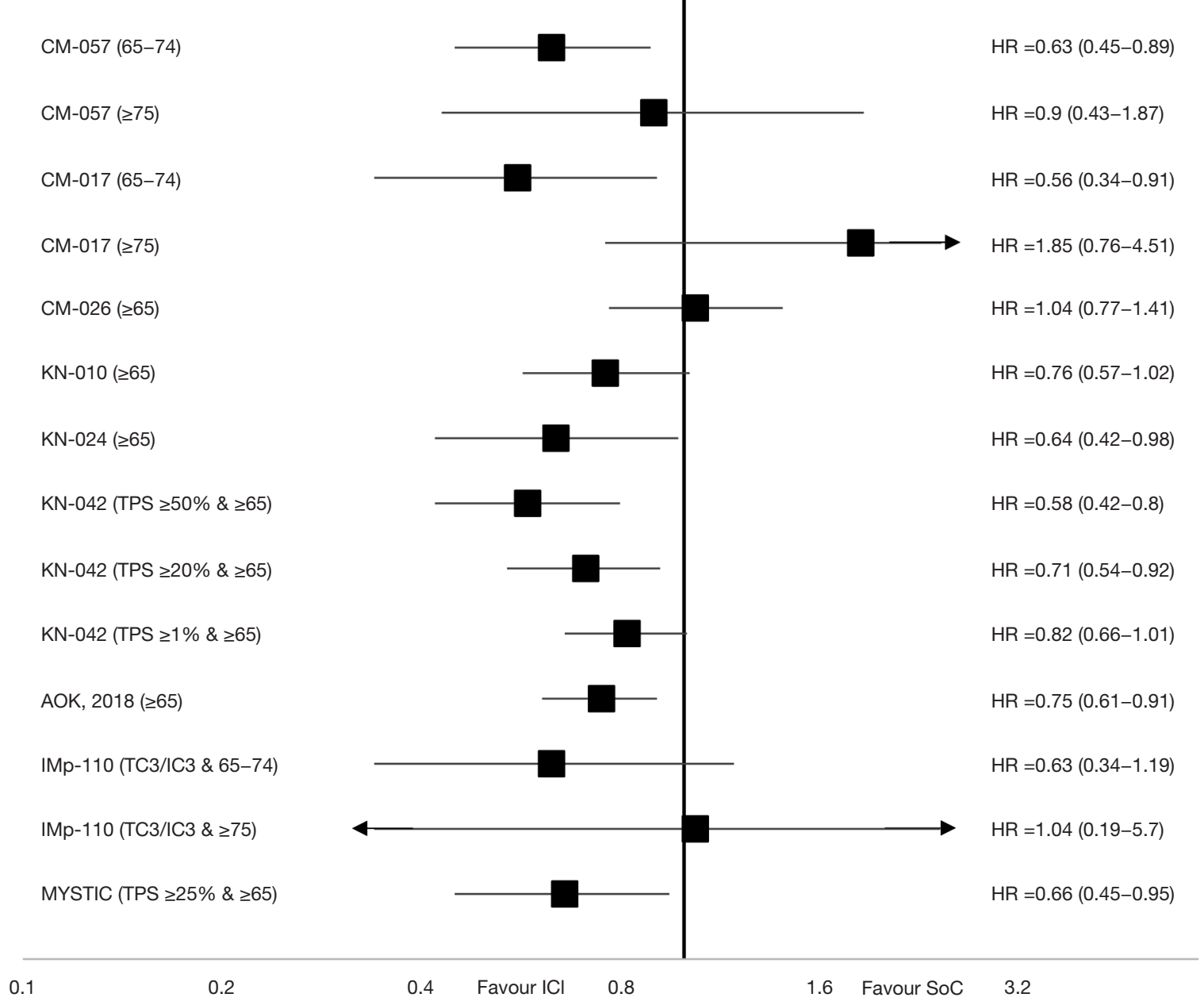

Figure $1 \mathrm{HR}$ for OS in elderly patients from phase III trials testing single agent anti-PD-1 or PD-L1 in aNSCLC. HR, hazard ratio; OS, overall survival; aNSCLC, advanced non-small cell lung cancer; ICI, immune checkpoint inhibitor; SoC, standard of care.

The phase III study CheckMate 026 investigated Nivolumab as monotherapy in untreated aNSCLC with PD-L1 tumor proportion score (TPS) $\geq 1 \%$. Patients were randomly assigned to Nivolumab $3 \mathrm{mg} / \mathrm{kg}$ every 2 weeks $(n=271)$ or platinum-based chemotherapy $(n=270)$ and evaluated on progression-free survival (PFS). Median age was 64 years and $11 \%(n=62)$ were at least 75 years. Nivolumab failed to improve PFS in overall population (HR, 1.15 ; $95 \%$ CI, 091 to 1.45 ) and in the subgroup of older patients ( $\geq 65$ years). OS was also in favour of chemotherapy in both populations (15).

Some studies with Nivolumab have been designed to investigate the efficacy and safety in these frail populations. The single arm phase II study CheckMate 171 enrolled 811 previously treated squamous aNSCLC with broad eligibility criteria to evaluate safety profile in frail populations. Thus, $34 \%(\mathrm{n}=278 / 811)$ of patients were at least 70 years and $15 \%(n=125 / 811) \geq 75$ years. This study showed similar survivals compared to the pivotal study with respectively mOS of 10.0 and 11.2 months for patients aged $\geq 70$ and $\geq 75$ years. The overall RR was $12.6 \%$ for patients aged $\geq 70$ years and $13.6 \%$ for those aged $\geq 75$ years, lower than in CheckMate 017 but could be due to an early tumor assessment (8/9 weeks) (16). CheckMate 153 was a phase IIIb/IV study of Nivolumab as monotherapy for previously treated aNSCLC leaving in the United States (US) or Canada. From April 2014 to May 2017, 1,426 patients were treated of whom 39\% $(\mathrm{n}=556 / 1,426)$ aged at least 70 years. The median OS was 9.1 months in the overall population and 10.3 months in 
aged population ( $\geq 70$ years) (17).

\section{Pembrolizumab}

Pembrolizumab is a humanised $\operatorname{IgG} 4 \mathrm{mAb}$ against PD-1. Drug development was rapidly restricted to aNSCLC with PD-L1-positive tumor expression. In Keynote-010, a phase II/III study, 1,033 previously treated patients with aNSCLC and a PD-L1 TPS $\geq 1 \%$ were randomized between Pembrolizumab $2 \mathrm{mg} / \mathrm{kg}$ ( $\mathrm{n}=344)$, Pembrolizumab $10 \mathrm{mg} / \mathrm{kg}(\mathrm{n}=346)$ or Docetaxel $75 \mathrm{mg} / \mathrm{m}^{2}(\mathrm{n}=343)$, all given every 3 weeks. The primary endpoint was OS, the median age was 63 years in both experimental arms versus 62 years in the control arm, $41.5 \%(n=429 / 1,033)$ were 65 years or older but no patient older than 70 years were enrolled in this trial. In the total population, Pembrolizumab improved OS in both experimental arms with mOS of 10.4 months (HR, $0.71 ; 95 \%$ CI, 0.58 to 0.88 ) for Pembrolizumab $2 \mathrm{mg} / \mathrm{kg}$ and 12.7 months (HR, $0.61 ; 95 \%$ CI, 0.49 to 0.75 ) for Pembrolizumab $10 \mathrm{mg} / \mathrm{kg}$ compared to 8.5 months for Docetaxel group. In a pooled analysis of both experimental arm, a non-statistically significant benefit was also observed for elderly population aged $\geq 65$ years (HR, $0.76 ; 95 \%$ CI, 0.57 to 1.02$)(10)$.

Moving to untreated patients with aNSCLC and high PD-L1 expression (TPS $\geq 50 \%$ ), in the phase III Keynote-024 study, single agent Pembrolizumab at fixed dose (200 mg every 3 weeks) was compared to platinumbased chemotherapy. This study enrolled three hundred five patients with PFS as primary endpoint. The median age was 64.5 years in Pembrolizumab group, 66 years in chemotherapy groups and $53.8 \%(n=164 / 305)$ were at least 65 years. In the overall population, pembrolizumab demonstrated an improvement on the PFS with a median PFS (mPFS) of 10.3 months for Pembrolizumab and 6.0 months for chemotherapy (HR, 0.50; 95\% CI, 0.37 to 0.68 ). This benefit was consistent across all subgroups included elderly population aged at least 65 years (HR, 0.45; $95 \%$ CI, 0.29 to 0.70 ) (11). An updated analysis confirmed this advantage prolonging mOS from 14.2 months for chemotherapy to 30.0 months for Pembrolizumab (HR, $0.63 ; 95 \% \mathrm{CI}, 0.47$ to 0.86 ). This benefit was also observed in population at least 65 years (HR, $0.64 ; 95 \% \mathrm{CI}, 0.42$ to 0.98) (18).

In the Keynote-042 study, 1,274 patients with untreated aNSCLC and a PD-L1 TPS at least $1 \%$ were randomly assigned between Pembrolizumab (200 mg fixed dose every 3 weeks) and a platinum-based chemotherapy. Despite a high proportion of patients with PD-L1 TPS >50\% ( $47 \%$ of patients), cross over was not allowed in this study and only $20 \%$ of patients in chemotherapy arm received subsequent immunotherapy. The primary endpoints were OS in different subgroups according to PD-L1 TPS ( $\geq 50 \%$, $\geq 20 \%$ and $\geq 1 \%$ ). The median age was 63 years in both arms, and $44.5 \%(n=567 / 1,274)$ were aged at least 65 years. In the overall population, Pembrolizumab significantly improved OS with a mOS of 20.0 versus 12.2 months (HR, $0.69 ; 95 \%$ CI, 0.56 to 0.85 ) in PD-L1 TPS $\geq 50 \%$ group, 17.7 versus 13 months (HR, 0.77 ; $95 \%$ CI, 0.64 to 0.92 ) for TPS $\geq 20 \%$ and 16.7 versus 12.1 months (HR, $0.81 ; 95 \%$ CI, 0.71 to 0.96 ) for PD-L1 TPS $\geq 1 \%$ group. In subgroup analysis, the benefit in elderly population ( $\geq 65$ years) was statistically significant for PD-L1 TPS $\geq 50 \%$ group (HR, $0.58 ; 95 \%$ CI, 0.42 to 0.80 ) and for PD-L1 TPS $\geq 20 \%$ group (HR, $0.71 ; 95 \% \mathrm{CI}, 0.54$ to 0.92 ) but not in the $\mathrm{PD}$ $\mathrm{L} 1 \geq 1 \%$ group (HR, $0.82 ; 95 \%$ CI, 0.66 to 1.01 ) (19).

A pooled-analysis of the Keynote-010, Keynote-024 and Keynote-042 studies evaluated efficacy of Pembrolizumab as single agent in 264 elderly patients aged at least 75 years. Pembrolizumab statistically improved OSs in PD-L1 TPS $\geq 50 \%$ elderly group $(\mathrm{n}=132, \mathrm{HR}, 0.40 ; 95 \% \mathrm{CI}, 0.25$ to 0.64 ) included untreated patients (HR, 0.41; 95\% CI, 0.23 to 0.73 ) compared to chemotherapy arm. In PD-L1 TPS $\geq 1 \%$ group, a non-statistical benefit was observed in favour of Pembrolizumab (HR, 0.76; 95\% CI, 0.56 to 1.02) (20).

\section{Atezolizumab}

Atezolizumab is an engineered, humanised IgG1 monoclonal anti-PD-L1 antibody able to block interactions between PD-L1 and PD-1 or B7.1. A companion test of Atezolizumab has been developed for the testing of PD-L1 expression on tumor cells (TC) and immune cells (IC). The POPLAR trial is an open-label, randomised phase II study evaluating Atezolizumab (1,200 mg fixed dose) compared to Docetaxel $\left(75 \mathrm{mg} / \mathrm{m}^{2}\right)$ every 3 weeks in previously treated aNSCLC with a stratification according to PD-L1 tumor and immune expression. Among the 287 patients enrolled, the median age was 62 years in both arms. Atezolizumab statistically improved OS in patients with PD-L1positive tumor expression compared to chemotherapy. Unfortunately, no aged-specific survival data were available in this trial (21).

In the phase III OAK study, 1,225 previously treated aNSCLC were randomly assigned to Atezolizumab $1,200 \mathrm{mg}$ or Docetaxel $\left(75 \mathrm{mg} / \mathrm{m}^{2}\right)$ every 3 weeks. The 
median age was 63.0 years in experimental group versus 64.0 years in control arm and 46\% $(n=564 / 1,225)$ were aged at least 65 years. In the first OS analysis after enrolment of 850 patients, Atezolizumab improved OS in the overall population with 13.8 versus 9.6 months in docetaxel group (HR, 0.73; 95\% CI, 0.62 to 0.87 ). This benefit was seen across all PD-L1 expression subgroups and in particular in elderly population (HR, 0.66; 95\% CI, 0.52 to 0.83 ) (22). The updated final analysis with 1,225 patients enrolled confirmed the benefit in the group of patient aged at least 65 years (HR, 0.75; 95\% CI, 0.61 to 0.91 ) (9).

The IMpower110 phase III trial evaluated single agent Atezolizumab in frontline versus platinum-based chemotherapy in PD-L1-positive aNSCLC. Among 554 patients enrolled (277 patients in each arm), half of patients were aged at least 65 years $(n=277 / 554)$ in the overall population and in the TC3/IC3 subgroup $(n=103 / 205)$. Atezolizumab significantly improved OS for patients with high-PD-L1 expression (TC3/IC3) with 20.2 versus 13.1 months for control arm (HR, 0.59; 95\% CI, 0.40 to 0.89 ). In subgroups analysis for patients with highPD-L1 expression, a non-significant benefit in favour of Atezolizumab was found for patient aged between 65 and 74 years (HR, 0.63 ; 95\% CI, 0.34 to 1.19 ) but no effect in the small sample of older patients (HR, 1.04; 95\% CI, 0.19 to 5.70$)$. No aged-specific survival data were available for $\mathrm{TC} 1 / 2 / 3$ or IC1/2/3 analysis (23).

\section{Durvalumab}

Durvalumab is a selective, fully humanised IgG1 antiPD-L1 mAb engineered to reduce antibody-dependent cell mediated cytotoxicity. Single arm phase II study (ATLANTIC) investigated Durvalumab as monotherapy in third-line or later treatment for aNSCLC in three distinct cohorts (cohort 1: patients with EGFR or ALK alteration; cohort 2: patients with wild type for EFGR and ALK; cohort 3: patients with PD-L1 TPS >90\%). The median age was respectively $61.0,62.0$ and 61.0 years for cohort 1 , 2 and 3. No patient older than 68 years has been included in this study. In the cohort 2, among patients with PDL1 TPS $\geq 25 \%$, objective response rate (ORR) was $20.3 \%$ [13/64] for patients aged at least 65 years versus $13.4 \%$ [11/82] in younger. In this same cohort for patients with PD-L1 TPS <25\%, ORR was lower for older patients $(2.7 \%$; $1 / 37)$ than younger $(10.7 \% ; 6 / 56)(24)$.

ARCTIC is a randomized phase III trial evaluating Durvalumab with or without the anti-CTLA4 antibody
Tremelimumab compared to the SoC for patients with aNSCLC after two systemic regimens. In the study A, 126 patients with PD-L1 TPS $\geq 25 \%$ were randomly assigned to received Durvalumab (10 $\mathrm{mg} / \mathrm{kg}$ every 2 weeks) or SoC. The median age was 63.5 years in Durvalumab arm, 62.0 years in SoC arm and 44\% [56/126] were aged at least 65 years. An improvement of mOS was observed in favour of Durvalumab with mOS of 11.7 versus 6.8 months (HR, $0.63 ; 95 \%$ CI, 0.42 to 0.93 ) but unfortunately, no aged-specific survival data were available (25).

The MYSTIC phase III randomized trial investigated Durvalumab alone or in combination with Tremelimumab compared to platinum-based chemotherapy in first-line for patients with aNSCLC. Among 488 patients with PD-L1 TPS $\geq 25 \%$, the median age was 64.0 years in Durvalumab arm, 64.5 years in chemotherapy arm and 49.8\% [162/325] was aged at least 65 years. One co-primary endpoint was OS and crossover was not allowed. Durvalumab did not significantly improve OS compared to chemotherapy in the overall population with 16.3 months for Durvalumab and 12.9 months for control arm (HR, 0.76; 95\% CI, 0.56 to 1.02). Interestingly, the benefit for Durvalumab was significant in subgroup of patients aged at least 65 years (HR, 0.66; 95\% CI, 0.45 to 0.95 ) (26).

\section{ICI efficacy in real-world studies}

Data based on real-world studies can provide a wider and longitudinal view of newly approved treatments in particular in under-represented subgroups of patients enrolled in pivotal studies as elderly population or patients with poor clinical conditions.

Grossi et al. analysed Nivolumab in elderly patients treated for squamous aNSCLC from the Italian expanded access program (EAP). On the 371 patients, $47 \%(n=175)$ were aged between 65 and 74 years and $19 \%(n=70)$ $\geq 75$ years with a higher proportion of smokers in the later groups compared to the overall population. Efficacy was considered equal in term of ORR, mOS, mPFS despite the same slight difference than in CheckMate 017 study in favour of younger population (<75 years) with respectively mOS of 5.8 versus 7.9 months and mPFS of 3.2 versus 4.2 months (27). Galli et al. led a monocentric retrospective analysis between April 2013 and March 2019 to collect data on elderly patients with aNSCLC treated with ICI. They identified 290 patients, 62\% $(n=180)$ younger than 70 years, $32 \%(n=94)$ aged between 70 and 79 years and $6 \%(n=16)$ at least 80 years. They did not observe 
any difference of efficacy in older population compared to younger with respectively a RR of $21.5 \%, 22.3 \%$ and $18.8 \%$ and a mOS of $9.1,11.3$ and 9.6 months for patients aged $<70$ years versus patients aged $70-79$ years versus patients $\geq 80$ years (28).

Juergens et al. collected data from the Canadian registry concerning Nivolumab given as compassionate use for aNSCLC. Among 472 patients identified, 13\% were at least 75 years. In the overall population, the mOS was 12.0 months and the median time to treatment discontinuation (TTD) was 3.5 months. No difference was seen across the subgroups according to the age (29). In another analysis, they focused on the Canadian cohort of patients from CheckMate 169 study, an EAP of Nivolumab for patients with previously treated aNSCLC. On 161 patients of whom 30\% aged at least 70 years, mOS was 9.1 in the overall population, compared to 8.0 months in the elderly population (30).

A retrospective analysis from the French national hospitals database compared Nivolumab outcomes between octogenarian and younger patients treated for aNSCLC. Among 10,452 patients, 4.9\% ( $\mathrm{n}=514)$ were aged at least 80 years with a median age of 82.5 years versus 62.8 years for the younger population. The survivals were similar between the two groups, especially for mOS with 11.5 months for elderly versus 11.6 months in younger patients (31).

Finally, a US retrospective study collected electronic health records from patients treated with ICI for an aNSCLC between January 2011 and March 2016. Among 1,344 patients identified, $27.2 \%$ were aged at least 75 years. The mOS in this latter group was 8.0 versus 7.5 months for younger patients. No difference has been found in term of survivals according to the age of patients (32).

\section{ICI as monotherapy from meta-analysis and pooled-analysis}

Some meta-analyses have investigated efficacy and safety profile of ICIs across different metastatic solid tumors. In 2018, Elias et al. conducted a meta-analysis to explore the efficacy of ICIs on elderly population based on nine randomised clinical trials in solid tumors including five studies in lung cancer. Among 5,458 patients from the nine studies, $42.57 \%$ were aged at least 65 years. In the overall population, PD-1/PD-L1 inhibitors demonstrated a $31 \%$ reduction in the hazard of death compared to SoC. This benefit was consistent across the subgroups with a HR of 0.68 (95\% CI, 0.61 to 0.75 ) for patients aged $<65$ years versus 0.64 (95\% CI, 0.54 to 0.76 ) for elderly population. No data were available for patients aged at least 75 years (33). Meta-analysis from Nishijima et al. confirmed these results with an analysis of nine studies for solid tumor including only two clinical trials focused on lung cancer. With an age cut-off of 65-70 years, they observed an OS improvement for ICI therapies compared to $\mathrm{SoC}$ in both younger (HR, 0.75; $95 \%$ CI, 0.68 to 0.82 ) and older (HR, $0.73 ; 95 \%$ CI, 0.62 to 0.87$)$ groups. However, in subgroup analysis of four anti-PD-1 therapies trials, they did not observe any OS improvement for patients aged $\geq 75$ years (HR, 0.86; $95 \%$ CI, 0.41 to 1.83 ). This result could be due to a heterogeneity of trials and to the small size of the elderly group (34). Landre et al. have also highlighted this latter result in their pooled-analysis of three studies in solid tumors evaluating Nivolumab as single agent (two studies in aNSCLC and one in metastatic renal cell carcinoma). In the subgroup of patients at least 75 years, the benefit from Nivolumab was uncertain (HR, 1.22; 95\% CI, 0.80 to 1.85$)$ compared to SoC (35).

Two recent analyses investigated this question especially in aNSCLC. Marur et al. evaluated OS survivals according to the age of patients from four clinical trials using ICIs in previously treated aNSCLC. Among 2,824 patients, $21.5 \%$ were aged $>70$ years and $12 \%$ aged $>75$ years. They concluded that the benefit from ICIs in patients aged 65 years or older was similar than younger patients with respectively mOS of $14.5,14.2$ and 14.7 months for patients younger than $65, \geq 65$ and $\geq 75$ years (36). The widest metaanalysis evaluating ICI's efficacy in elderly in lung cancer was conducted by Zhang et al. They selected 12 studies with 8,176 patients, including $46 \%(n=3,730)$ aged at least 65 years. Using a cut-off of 65 years, ICIs improved OS compared to SoC in both younger (HR, 0.75; 95\% CI, 0.65 to 0.87 ) and older (HR, $0.81 ; 95 \% \mathrm{CI}, 0.72$ to 0.92 ) groups. However, after a new subdivision of older group based on 75 years cut-off, compared to SoC, immunotherapy failed to demonstrate a statistical benefit in patients aged at least 75 years (HR, $0.90 ; 95 \%$ CI, 0.64 to 1.25 ) (37).

\section{Efficacy of ICI in combination with chemotherapy}

If chemotherapy might theoretically enhance the ICI activity through different mechanisms as the release of neoantigens or inhibition protumoral IC, the combination might be more toxic than monotherapy in particular 
in elderly patients. Combo ICI + platinum-based chemotherapy has been investigated in untreated patients with aNSCLC regardless PD-L1 expression (Table 2).

\section{Pembrolizumab}

In the double-blind phase III Keynote-189 study, untreated patients with non-squamous aNSCLC were randomly assigned (in a 2:1 ratio) to receive Pemetrexed + platinumbased drug and Pembrolizumab (200 $\mathrm{mg}$ fixed dose) or placebo. Among 616 enrolled patients, the median age was 65 years in the experimental arm versus 63.5 years in the control arm and $52 \%$ were aged at least 65 years. In the overall population, Pembrolizumab combination dramatically improved both OS (HR, 0.49; 95\% CI, 0.38 to 0.64 ) and PFS (HR, 0.52; 95\% CI, 0.43 to 0.64 ). In the subgroup of elderly patients ( $\geq 65$ years), a significant OS improvement in favour of the Pembrolizumab combination (HR, $0.52 ; 95 \%$ CI, 0.43 to 0.64 ) was found, but not for the PFS (HR, 0.75 ; $95 \%$ CI, 0.55 to 1.02 ) (38). In the phase III Keynote-407 study, untreated squamous aNSCLC were randomly assigned to receive Carboplatin and a taxane drug with Pembrolizumab or placebo. On 559 patients included, the median age was 65 years in both arms and $54.5 \%$ were at least 65 years. Combo ICI + chemotherapy significantly increased OS (HR, $0.64 ; 95 \%$ CI, 0.49 to 0.85 ) with mOS of 15.9 months for experimental arm versus 11.3 months for SoC. However, in subgroup analysis of elderly patients ( $\geq 65$ years), the benefit was uncertain (HR, 0.74; $95 \%$ CI, 0.51 to 1.07) (39). For these two phase III studies, no specific-aged survivals data were available for the subgroup of patients aged at least 70 years.

\section{Atezolizumab}

In the phase III IMpower-130 study, untreated nonsquamous aNSCLC were randomly assigned (in a 2:1 ratio) to receive chemotherapy (carboplatin + taxane drug) with or without Atezolizumab (1,200 $\mathrm{mg}$ fixed dose). Among 724 patients, the median age was 64 years in Atezolizumab combination arm versus 65 years in control arm, $38 \%$ $(\mathrm{n}=276)$ were aged between 65 and 74 and $11 \%(\mathrm{n}=82)$ aged at least 75 years. Adjunction of Atezolizumab to chemotherapy improved OS compared to control arm with 18.6 versus 13.9 months in favour of Atezolizumab combination (HR, $0.79 ; 95 \%$ CI, 0.64 to 0.98 ) in the overall wild-type population but not in the subgroup of patients aged at least 65 years (HR, 0.78 ; $95 \%$ CI, 0.58 to 1.05 ).
Concerning PFS, benefit for Atezolizumab combination was seen in the overall population and across all subgroups according to the age (40). Interestingly, the phase III IMpower150 study investigated the role of bevacizumab in combination of chemotherapy and Atezolizumab. In the wild-type population, 1,040 untreated non-squamous aNSCLC were randomly assigned to receive Atezolizumab plus Carboplatin plus Paclitaxel (ACP), Bevacizumab plus Carboplatin plus Paclitaxel (BCP) or Atezolizumab plus Bevacizumab plus Carboplatin plus Paclitaxel (ABCP). In first analysis, $\mathrm{ABCP}$ was compared with the $\mathrm{BCP}$ group according the PFS. Among 800 patients enrolled in these two latter groups, the median age was 63 years in both groups and respectively $35 \%(n=281), 9 \%(n=72)$ and $<1 \%(\mathrm{n}=6)$ were aged $65-74,75-84$ and $\geq 85$ years. ABCP treatment improved PFS compared to BCP group with mPFS of 8.3 versus 6.8 months in favour of $\mathrm{ABCP}$ group (HR, $0.62 ; 95 \% \mathrm{CI}, 0.52$ to 0.74 ). In subgroup analysis, this benefit is confirmed in younger ( $<65$ years) and intermediate (65-74 years) patients but uncertain for patients at least 75 years (41).

\section{Nivolumab}

In the wide multi-arm phase III CheckMate 227 study, the part 2 study especially investigated the impact of addition of Nivolumab to chemotherapy in untreated non-squamous aNSCLC with PD-L1 TPS $<1 \%$. Patients were randomly assigned to receive chemotherapy with or without Nivolumab (360 fixed dose every 3 weeks). Among 543 included patients, the median age was 63 years in both arms and respectively $35 \%(n=190)$ and $9 \%(n=49)$ were aged 65 to 74 or $\geq 75$ years. This study did not meet the primary endpoint, neither in the overall population (HR, 0.86; 95\% CI, 0.69 to 1.08), nor subgroups based on age, in particular in patients aged at least 75 where the combination seemed to be deleterious (HR, 1.21) (42).

\section{Efficacy of combination of ICI}

If combinations ICI plus chemotherapy became the Soc for untreated aNSCLC, an option appeared with the combination of anti-PD-1/PD-L1 and anti-CTLA4.

In the phase III CheckMate 227 study, patients with untreated aNSCLC were randomly assigned according to PD-L1 expression to received Nivolumab versus Nivolumab plus Ipilimumab versus platinum-based chemotherapy in case of PD-L1-positive tumor expression or Nivolumab 
Table 2 Phase III studies of anti-PD-1 or anti-PD-L1 mAbs in combination with chemotherapy or anti-CTLA4 mAbs in aNSCLC with subgroup analysis data in the elderly population

\begin{tabular}{|c|c|c|c|c|c|c|c|c|c|c|c|c|}
\hline \multirow{2}{*}{ Author (year) } & \multirow{2}{*}{ Study } & \multirow{2}{*}{ Phase } & \multirow{2}{*}{ Histology } & \multirow{2}{*}{ Line } & \multirow{2}{*}{ Treatment arms } & \multirow{2}{*}{ No. of patients } & \multirow{2}{*}{ Median age (years) } & \multirow{2}{*}{$\begin{array}{l}\text { Age cut-off, older } \\
\text { patients }\end{array}$} & \multirow{2}{*}{ No. of older [\%] } & \multicolumn{3}{|c|}{ Overall survivals } \\
\hline & & & & & & & & & & Overall HR $(95 \% \mathrm{Cl})$ & HR for younger $(95 \% \mathrm{Cl})$ & HR for older $(95 \% \mathrm{Cl})$ \\
\hline Gandhi (2018) & Keynote-189 & III & Nonsquamous NSCLC & 1 & $\begin{array}{l}\text { Carbo + Pem + Pembro vs. } \\
\text { Carbo + Pem + PCB }\end{array}$ & 616 & 65.0 [34-84]; $63.5[34-84]$ & $\geq 65$ & 304 [49] & $0.49(0.38-0.64)$ & $0.43(0.31-0.61)$ & $0.64(0.43-0.95)$ \\
\hline Paz-Ares (2018) & Keynote-407 & III & Squamous NSCLC & 1 & $\begin{array}{l}\text { Carbo + (nab)Pacli + Pembro vs. } \\
\text { Carbo + (nabPacli + PCB }\end{array}$ & 559 & 65.0 [29-87]; $65.0[36-88]$ & $\geq 65$ & $305[55]$ & $0.64(0.49-0.85)$ & $0.52(0.34-0.80)$ & $0.74(0.51-1.07)$ \\
\hline West (2019) & IMpower-130 & III & Nonsquamous NSCLC & 1 & $\begin{array}{l}\text { Carbo + (nab)Pacli + Atezo vs. } \\
\text { Carbo + (nabPacli + PCB }\end{array}$ & 723 & 64.0 [18-86]; $65.0[$ [38-85] & $\geq 65: 65-74 ; \geq 75$ & 361 [50]: 276 [38]; 85 [12] & $0.79(0.64-0.98)$ & $\begin{array}{l}0.79(0.58-1.08) ; \\
\text { WT. pop } \geq 65\end{array}$ & $\begin{array}{l}0.78 \text { (0.58-1.05); } \\
\text { WT. pop \65 }\end{array}$ \\
\hline Socinski (2018) & IMpower-150 & IIII & Nonsquamous NSCLC & 1 & $\begin{array}{c}\text { Carbo + Pacli + Atezo vs. Carbo + Pacli + } \\
\text { Beva vs. Carbo + Pacli + Beva + Atezo }\end{array}$ & 1,202 & $\mathrm{NA} ; 63.0$ [31-90]; 63.0 [31-89] & $\geq 65: 65-74 ; \geq 75$ & 359 [45]: 281 [35]; 78 [10] (on 800) & $0.78(0.64-0.96)$ & NA & NA \\
\hline Paz-Ares (2019) & CheckMate 227 & III & Nonsquamous NSCLC & 1 & Plat + Pem + Nivo vs. Plat + Pem & 543 & $63.0[27-84] ; 64.0[31-83]$ & $\geq 65: 65-74 ; \geq 75$ & 239 [44]: 190 [35]; 49 [9] & $0.86(0.69-1.08)$ & $0.76(\mathrm{NA})$ & $0.95(\mathrm{NA}) ; 1.21(\mathrm{NA})$ \\
\hline Reck (2020) & CheckMate 9LA & III & NSCLC & 1 & Nivo + Ipi + Chemo (2cycles) vs. Chemo & 719 & $65.0[35-81] ; 65.0[26-86]$ & $\geq 65: 65-74 ; \geq 75$ & 365 [51]: 295 [41]; 70 [10] & $0.66(0.55-0.80)$ & $0.61(\mathrm{NA})$ & $0.62(\mathrm{NA}) ; 1.21$ (NA) \\
\hline Hellman (2019) & CheckMate 227 & III & NSCLC & 1 & Nivo + Ipi vs. PBCh & 1,166 Ov pop & 64.0 [26-87]; $64.0[29-87]$ & $\geq 65 ; 65-74 ; \geq 75$ & 556 [48]: 442 [38]; 113 [10] & $0.73(0.64-0.84)$ & $0.70(0.58-0.85)$ & $\begin{array}{l}0.76(0.61-0.95) \\
0.84(0.55-1.29)\end{array}$ \\
\hline Hellman (2019) & CheckMate 227 & III & NSCLC & 1 & Nivo + Ipi vs. PBCh & 793 PD-L1+ & $64.0[26-84] ; 64.0[29-87]$ & $\geq 65: 65-74 ; \geq 75$ & 387 [49]: 306 [39]; 81 [10] & $0.79(0.65-0.96)$ & $0.70(0.55-0.89)$ & $\begin{array}{l}0.91(0.70-1.19) \\
0.92(0.57-1.48)\end{array}$ \\
\hline Hellman (2019) & CheckMate 227 & III & NSCLC & 1 & Nivo + Ipi vs. PBCh & 373 PD-L1- & $63.0[34-87] ; 64.0[30-80]$ & $\geq 65: 65-74 ; \geq 75$ & 168 [45]: 136 [36]; 32 [9] & $0.62(0.48-0.78)$ & $0.69(0.50-0.94)$ & $\begin{array}{l}0.49(0.32-0.75) ; \\
0.75(0.31-1.82)\end{array}$ \\
\hline Planchard (2020) & ARCTIC Study B & III & NSCLC & $>2$ & $\begin{array}{l}\text { Durva + Treme vs. Durva vs. } \\
\text { Treme vs. Soc }\end{array}$ & 469 & $\begin{array}{l}62.5 \text { [26-81]; } 63.0[19-83] ; \\
63.5[45-81] ; 65.0[42-83]\end{array}$ & $\geq 65$ & $221[47]$ & $\begin{array}{l}0.80 \text { (0.61-1.05); } \\
0.80 \text { (0.59-1.08); } \\
1.02 \text { (0.71-1.46) }\end{array}$ & NA & NA \\
\hline
\end{tabular}

Dilimumab; PBCh, platinum-based chemotherapy; Durva, Durvalumab: Treme, Tremelimumab; SoC, standard of care; NA, not available; Ov pop, overall population; WT. pop, wild-type population. 
plus Ipilimumab versus Nivolumab plus platinum-based chemotherapy versus platinum-based chemotherapy in case of PD-L1-negative tumor expression. For the efficacy analysis of Nivolumab plus Ipilimumab arm, 1,166 were enrolled with a median age of 64 years, $38 \%(n=442)$ of patients aged between 65 to 74 years and $9.7 \%(n=113)$ patients aged at least 75 years. In the overall population ICIs combination improved OS regardless PD-L1 expression, but in the subgroup of elderly population ( $\geq 75$ years) the benefit was unclear in PD-L1-positive tumor (HR, 0.92; $95 \%$ CI, 0.57 to 1.48 ) as well as PD-L1 negative tumor (HR, 0.75 ; $95 \%$ CI, 0.31 to 1.82 ) (43).

In the two phase III studies evaluating Durvalumab in combination with Tremelimumab, either in previously treated aNSCLC (ARCTIC study) or in untreated patients (MYSTIC study), specific-aged survival data are lacking. The both two studies failed to demonstrate an OS improvement in favour of ICI combination $(25,26)$.

Recently, results from the phase III CheckMate 9LA study have been presented at ASCO congress. This trial investigated combination of Nivolumab plus Ipilimumab plus two initial cycles of platinum-based chemotherapy compared to platinum-based chemotherapy in untreated aNSCLC. This trial enrolled 719 patients with a median age of 65 years in both arms, $41 \%(n=298)$ aged 65 to 74 years and $9.7 \%(n=70)$ aged $\geq 75$ years. Combination of Nivolumab plus Ipilimumab plus early chemotherapy improved OS with mOS of 15.6 versus 10.9 month in favour of experimental arm (HR, 0.66; 95\% CI, 0.55 to 0.80 ). In subgroup analysis, elderly population ( $\geq 75$ years) seemed to not derive a benefit from this experimental arm (HR, 1.21) (44).

\section{ICI efficacy in ES-SCLC}

In ES-SCLC, ICI has been firstly investigated as monotherapy with the hope to change the prognostic of this aggressive disease. Unfortunately, after initial promising results, ICI as single agent has been largely disappointing. However, reflecting the change of SoC in aNSCLC, combination chemotherapy plus ICI has been evaluated in untreated ES-SCLC and modified the clinical practice. First, IMpower133 tested combination Carboplatin plus etoposide with or without Atezolizumab $(1,200 \mathrm{mg}$ fixed dose) in first-line treatment for ES-SCLC. This trial enrolled 403 patients with a median age of 64 years in both arms and $46 \%(n=186)$ of patients aged $\geq 65$ years. Combination chemotherapy plus Atezolizumab improved OS compared SoC with a mOS of 12.3 versus 10.3 months in favour of experimental arm (HR, 0.70; 95\% CI, 0.54 to 0.91 ). Curiously, subgroup analysis showed a stronger benefit in older (HR, $0.53 ; 95 \% \mathrm{CI}, 0.36$ to 0.77 ) than younger patients (HR, $0.92 ; 95 \% \mathrm{CI}, 0.64$ to 1.32 ) (45). The second study in the same setting was the phase III CASPIAN study testing Durvalumab with or without Tremelimumab in combination with platinum-etoposide regimen. Among the 537 patients enrolled in two arms of chemotherapy with or without Durvalumab, the median age was 62 years for experimental arm versus 63 years in control arm and $39.7 \%(n=213)$ were aged at least 65 years. In the overall population, Durvalumab plus chemotherapy combination improved OS compared to Soc with a benefit of 2.7 months (HR, 0.73 ; 95\% CI, 0.59 to 0.91 ). In subgroup analysis, this benefit was not significant in elderly population (HR, 0.75 ; 95\% CI, 0.54 to 1.06 ) (46).

\section{Safety profile}

Safety profile of ICIs among elderly population is another key question. Immune-related adverse events (irAE) are induced by activated $\mathrm{T}$ cells infiltrated in normal tissue. If ICIs as single agent demonstrated a very good safety profile in overall population with less than $10 \%$ of severe side effects, these toxicities which can affect multiple organs, might be more difficult to manage in frail and elderly population. Despite the same spectrum of toxicities, antiCTLA4 is more toxic than anti-PD-1/PD-L1 with a higher frequency of severe toxicities (47). Across all pivotal studies evaluating anti-PD-1/PD-L1 as monotherapy, none of them reported a worse safety profile in elderly population. Thus, the FDA analysis of survival in older adults with aNSCLC in randomized trial found a lower proportion of grade 1$4 \mathrm{irAE}$ in patients at least 75 years $(15.1 \%)$ compared to patient $<65$ years (30.4\%) (36). Nosaki et al. in their pooled analysis of Pembrolizumab showed a better safety profile for ICI than chemotherapy in elderly population (20). In the CheckMate 171 real-world study, safety profile was similar between younger and older patients except for low-grade diarrhoea which was more frequent in elderly population (16). In CheckMate 153, the incidence of highgrade treatment-related adverse events (TRAEs) was similar across all subgroups with respectively $6 \%$ of TRAEs in both overall population and elderly group (17). Unfortunately, aged-specific safety data are not available at this time concerning trials with combination chemotherapy plus ICIs or combination of ICIs. 
In a meta-analysis of fatal toxic effects related to ICIs, Wang et al. observed that patients who died due to ICI side effect were older than those without fatal side effect (median age of 70 versus 62 years). It might be due to complications of hospitalization or complications of immunosuppressive agents used to treat irAE (48).

\section{Discussion}

Over the past 5 years, immunotherapy has seen a dramatic breakthrough in the lung cancer treatment. ICIs are now an essential treatment for a large part of patients as a single agent or in combination with chemotherapy. Derived from many robust phase III studies, results demonstrated an important survival benefit in favour of ICIs in the overall population. However, the population enrolled in these clinical trials is clearly younger than this met in our dailypractice and it is difficult to extrapolate these results in elderly population.

As monotherapy, older patients seem to derive the same benefit from ICIs than younger patients with no excess of toxicities but some limitations have to be noted. First, age cut-off for definition of elderly population is different across the clinical trials, some studies using 65 years reflecting World Health Organization definition and others analysing subgroups aged at least 70 or 75 years. The cut-off of 65 years increasing the number of aged patients enrolled in clinical trials leads to an important heterogeneity of this subgroup. Heterogeneity of the aged population is driven by a high variability of physiological age reflecting the co-morbidities, organs dysfunction or co-medications with a higher prevalence in the older population than younger. Some clinical parameters can also introduce a misinterpretation of ICIs outcomes. For instance, high performance status or sarcopenia represent two predictive and prognostic factors of poor survivals in particular for patients treated with ICIs even in younger population $(49,50)$. These clinical parameters are more frequent in the elderly population (51). Immunosenescence more likely associated with aging can also negatively impact outcomes of ICIs (12). Finally, a retrospective analysis demonstrated that aging was an independent risk factor of disease hyperprogression for patient treated with ICIs across solid tumor (52).

In combination with chemotherapy, impact of ICIs in elderly population is more doubtful. First, available data are extremely limited in the subgroup of aged patients. However, EMA have signalled according unpublished data that upon the 57 aged-patients ( $\geq 75$ years) from
Keynote-189, combination seems to have deleterious effect on OS (HR, 2.09; 95\% CI, 0.84 to 5.23) and PFS (HR, $1.73 ; 95 \%$ CI, 0.77 to 3.90 ) (53) compared to chemotherapy alone. These results although limited, should lead to use the combination in elderly patients with caution. Second, clinical studies evaluating combination of chemotherapy and ICI did not investigate the Carboplatin and weekly Paclitaxel regimen that represents the first option for treatment of aNSCLC in elderly patients (54).

Dedicated studies evaluating ICIs as single agent or in combination in elderly patients are currently on going. A mono-arm phase II study is evaluating the efficacy, safety and quality of life in aged patients ( $\geq 70$ years) treated with Pembrolizumab as monotherapy in first line for a PD-L1-positive aNSCLC (TPS $\geq 1 \%$ ) (55). The results from the randomized phase III ELDERLY Trial evaluating Carboplatin and weekly Paclitaxel regimens with or without Atezolizumab in 500 elderly patients with untreated aNSCLC will be awaited (56). This trial will clarify the place of combination of ICI plus chemotherapy for the management of elderly patients with aNSCLC. Two others phase II studies will complete data about ICI plus chemotherapy combination in aged patient, one investigating Pembrolizumab plus Pemetrexed in first line aNSCLC with TPS $<50 \%$ (57) and the other a doublet regimen (Carboplatin + Paclitaxel or Pemetrexed every 3 weeks) with Durvalumab (58). These two latter trials will have a lower impact than ELDERLY study due to smaller populations (50 and 41 patients expected). Finally, eNERGY trial (59), a randomized phase III trial testing Nivolumab + Ipilimumab versus platinum-based doublet regimen in two specific untreated aNSCLC populations (patient aged at least 70 years or with a poor performance status) is ongoing in France. This study should answer about the efficacy and safety profile of this combination in elderly patients.

In conclusion, dedicated studies are urgently needed to clarify the effect of ICIs in the elderly population in particular, since combinations chemotherapy and ICI have become the new SoC of first line treatment in aNSCLC and ES-SCLC.

\section{Acknowledgments}

Funding: None.

\section{Footnote}

Provenance and Peer Review: This article was commissioned 
by the editorial office, Translational Lung Cancer Research for the series "Immunotherapy in other thoracic malignancies and uncommon populations". The article has undergone external peer review.

Reporting Checklist: The authors have completed the Narrative Review reporting checklist. Available at http:// dx.doi.org/10.21037/tlcr-20-1239

Peer Review File: Available at http://dx.doi.org/10.21037/ tlcr-20-1239

Conflicts of Interest: Both authors have completed the ICMJE uniform disclosure form (available at http://dx.doi. org/10.21037/tlcr-20-1239). The series "Immunotherapy in other thoracic malignancies and uncommon populations" was commissioned by the editorial office without any funding or sponsorship. BB served as the unpaid Guest Editor of the series. $\mathrm{CN}$ reports personal fees from ASTRA ZENECA, personal fees from MSD, personal fees, nonfinancial support and other from ROCHE, non-financial support and other from AMGEN, non-financial support and other from BMS, from TAKEDA, other from PFIZER, outside the submitted work. BB reports grants from 4D Pharma, grants from Abbvie, grants from Amgen, grants from Aptitude Health, grants from AstraZeneca, grants from BeiGene, from Blueprint Medicines, grants from BMS, grants from Boehringer Ingelheim, grants from Celgene, grants from Cergentis, grants from Cristal Therapeutics, grants from Daiichi-Sankyo, grants from Eli Lilly, grants from GSK, grants from Inivata, grants from Janssen, grants from Onxeo, grants from OSE immunotherapeutics, grants from Pfizer, grants from Roche-Genentech, grants from Sanofi, grants from Takeda, grants from Tolero Pharmaceuticals, outside the submitted work. The authors have no other conflicts of interest to declare.

Ethical Statement: The authors are accountable for all aspects of the work in ensuring that questions related to the accuracy or integrity of any part of the work are appropriately investigated and resolved

Open Access Statement: This is an Open Access article distributed in accordance with the Creative Commons Attribution-NonCommercial-NoDerivs 4.0 International License (CC BY-NC-ND 4.0), which permits the noncommercial replication and distribution of the article with the strict proviso that no changes or edits are made and the original work is properly cited (including links to both the formal publication through the relevant DOI and the license). See: https://creativecommons.org/licenses/by-nc-nd/4.0/.

\section{References}

1. Available online: https://seer.cancer.gov/statfacts/html/ lungb.html

2. Owonikoko TK, Ragin CC, Belani CP, et al. Lung cancer in elderly patients: an analysis of the surveillance, epidemiology, and end results database. J Clin Oncol 2007;25:5570-7.

3. Singh H, Kanapuru B, Smith C, et al. FDA analysis of enrollment of older adults in clinical trials for cancer drug registration: A 10-year experience by the U.S. Food and Drug Administration. J Clin Oncol 2017;3:abstr 10009.

4. Sacher AG, Le LW, Leighl NB, et al. Elderly patients with advanced NSCLC in phase III clinical trials: are the elderly excluded from practice-changing trials in advanced NSCLC? J Thorac Oncol 2013;8:366-8.

5. Takayuki N, Keiko T, Junji U, et al. Advanced NonSmall-Cell Lung Cancer in Elderly Patients: Patient Features and Therapeutic Management. BioMed Res Int 2018;2018:8202971.

6. Elias R, Odejide O. Immunotherapy in Older Adults: A Checkpoint to Palliation?. Am Soc Clin Oncol Educ Book 2019;39:e110-20.

7. Brahmer J, Reckamp KL, Baas P, et al. Nivolumab versus Docetaxel in Advanced Squamous-Cell Non-Small-Cell Lung Cancer. N Engl J Med 2015;373:123-35.

8. Borghaei H, Paz-Ares L, Horn L, et al. Nivolumab versus Docetaxel in Advanced Nonsquamous Non-Small-Cell Lung Cancer. N Engl J Med 2015;373:1627-39.

9. Fehrenbacher L, von Pawel J, Park K, et al. Updated Efficacy Analysis Including Secondary Population Results for OAK: A Randomized Phase III Study of Atezolizumab versus Docetaxel in Patients with Previously Treated Advanced Non-Small Cell Lung Cancer. J Thorac Oncol 2018;13:1156-70. Erratum in: J Thorac Oncol. 2018 Nov;13(11):1800. doi: 10.1016/j.jtho.2018.08.003.

10. Herbst RS, Baas P, Kim DW, et al. Pembrolizumab versus docetaxel for previously treated, PD-L1-positive, advanced non-small-cell lung cancer (KEYNOTE-010): a randomised controlled trial. Lancet 2016;387:1540-50.

11. Reck M, Rodríguez-Abreu D, Robinson AG, et al. Pembrolizumab versus Chemotherapy for PD-L1Positive Non-Small-Cell Lung Cancer. N Engl J Med 
2016;375:1823-33.

12. Ferrara R, Mezquita L, Auclin E, et al. Immunosenescence and immunecheckpoint inhibitors in non-small cell lung cancer patients: Does age really matter? Cancer Treat Rev 2017;60:60-8.

13. Rizvi NA, Mazières J, Planchard D, et al. Activity and safety of nivolumab, an anti-PD-1 immune checkpoint inhibitor, for patients with advanced, refractory squamous non-small-cell lung cancer (CheckMate 063): a phase 2, single-arm trial. Lancet Oncol 2015;16:257-65.

14. Wu YL, Lu S, Cheng Y, et al. Nivolumab Versus Docetaxel in a Predominantly Chinese Patient Population with Previously Treated Advanced NSCLC: CheckMate 078 Randomized Phase III Clinical Trial. J Thorac Oncol 2019;14:867-75.

15. Carbone DP, Reck M, Paz-Ares L, et al. First-Line Nivolumab in Stage IV or Recurrent Non-Small-Cell Lung Cancer. N Engl J Med 22 2017;376:2415-26.

16. Felip E, Ardizzoni A, Ciuleanu T, et al. CheckMate 171: A phase 2 trial of nivolumab in patients with previously treated advanced squamous non-small cell lung cancer, including ECOG PS 2 and elderly populations. Eur J Cancer 2020;127:160-72.

17. Spigel DR, McCleod M, Jotte RM, et al. Safety, Efficacy, and Patient-Reported Health-Related Quality of Life and Symptom Burden with Nivolumab in Patients with Advanced Non-Small Cell Lung Cancer, Including Patients Aged 70 Years or Older or with Poor Performance Status (CheckMate 153). J Thorac Oncol 2019;14:1628-39.

18. Reck M, Rodríguez-Abreu D, Robinson AG, et al. Updated Analysis of KEYNOTE-024: Pembrolizumab Versus Platinum-Based Chemotherapy for Advanced NonSmall-Cell Lung Cancer With PD-L1 Tumor Proportion Score of 50\% or Greater. J Clin Oncol 2019;37:537-46.

19. Mok TSK, Wu YL, Kudaba I, et al. Pembrolizumab versus chemotherapy for previously untreated, PD-L1expressing, locally advanced or metastatic non-small-cell lung cancer (KEYNOTE-042): a randomised, open-label, controlled, phase 3 trial. Lancet 2019;393:1819-30.

20. Nosaki K, Saka H, Hosomi Y, et al. Safety and efficacy of pembrolizumab monotherapy in elderly patients with PDL1-positive advanced non-small-cell lung cancer: Pooled analysis from the KEYNOTE-010, KEYNOTE-024, and KEYNOTE-042 studies. Lung Cancer 2019;135:188-95.

21. Fehrenbacher L, Spira A, Ballinger M, et al. Atezolizumab versus docetaxel for patients with previously treated non-small-cell lung cancer (POPLAR): a multicentre, open-label, phase 2 randomised controlled trial. Lancet
2016;387:1837-46.

22. Rittmeyer A, Barlesi F, Waterkamp D, et al. Atezolizumab versus docetaxel in patients with previously treated non-small-cell lung cancer (OAK): a phase 3, openlabel, multicentre randomised controlled trial. Lancet 2017;389:255-65.

23. Spigel D, de Marinis F, Giaccone G, et al. IMpower110: Interim overall survival (OS) analysis of a phase III study of atezolizumab (atezo) vs platinum-based chemotherapy (chemo) as first-line (1L) treatment (tx) in PD-L1-selected NSCLC. Ann Oncol 2019;30:V915.

24. Garassino MC, Cho BC, Kim JH, et al. Durvalumab as third-line or later treatment for advanced non-small-cell lung cancer (ATLANTIC): an open-label, single-arm, phase 2 study. Lancet Oncol 2018;19:521-36.

25. Planchard D, Yokoi T, McCleod MJ, et al. A Phase III Study of Durvalumab (MEDI4736) with or without Tremelimumab for Previously Treated Patients with Advanced NSCLC: Rationale and Protocol Design of the ARCTIC Study. Clin Lung Cancer 2016;17:232-236.e1.

26. Rizvi NA, Cho BC, Reinmuth N, et al. Durvalumab With or Without Tremelimumab vs Standard Chemotherapy in First-line Treatment of Metastatic Non-Small Cell Lung Cancer: The MYSTIC Phase 3 Randomized Clinical Trial. JAMA Oncol 2020;6:661-74.

27. Grossi F, Crinò L, Logroscino A, et al. Use of nivolumab in elderly patients with advanced squamous non-small-cell lung cancer: results from the Italian cohort of an expanded access programme. Eur J Cancer 2018;100:126-34.

28. Galli G, De Toma A, Pagani F, et al. Efficacy and safety of immunotherapy in elderly patients with non-small cell lung cancer. Lung Cancer 2019;137:38-42.

29. Juergens RA, Mariano C, Jolivet J, et al. Real-world benefit of nivolumab in a Canadian non-small-cell lung cancer cohort. Curr Oncol 2018;25:384-92.

30. Juergens R, Chu Q, Rothenstein J, et al. P2.07-029 CheckMate 169: Safety/Efficacy of Nivolumab in Canadian Pretreated Advanced NSCLC (including Elderly and PS 2) Patients. J Thorac Oncol 2017;12:S2426-7.

31. Assié J, Cotté F, Levra MG, et al. P2.04-03 Nivolumab Outcomes in Octogenarian Patients with Advanced NonSmall Cell Lung Cancer in French Real-World Setting. J Thorac Oncol 2019;14:S708.

32. Khozin S, Carson KR, Zhi J, et al. Real-World Outcomes of Patients with Metastatic Non-Small Cell Lung Cancer Treated with Programmed Cell Death Protein 1 Inhibitors in the Year Following U.S. Regulatory Approval. Oncologist 2019;24:648-56. 
33. Elias R, Giobbie-Hurder A, McCleary NJ, et al. Efficacy of PD-1 \& PD-L1 inhibitors in older adults: a metaanalysis. J Immunother Cancer 2018;6:26.

34. Nishijima TF, Muss HB, Shachar SS, et al. Comparison of efficacy of immune checkpoint inhibitors (ICIs) between younger and older patients: A systematic review and metaanalysis. Cancer Treat Rev 2016;45:30-7.

35. Landre T, Taleb C, Nicolas $\mathrm{P}$, et al. Is there a clinical benefit of anti-PD-1 in patients older than 75 years with previously treated solid tumour? J Clin Oncol 2016;34:abstr 3070.

36. Marur S, Singh H, Mishra-Kalyani P, et al. FDA analyses of survival in older adults with metastatic non-small cell lung cancer in controlled trials of PD-1/PD-L1 blocking antibodies. Semin Oncol 2018;45:220-5.

37. Zhang L, Sun L, Yu J, et al. Comparison of Immune Checkpoint Inhibitors between Older and Younger Patients with Advanced or Metastatic Lung Cancer: A Systematic Review and Meta-Analysis. BioMed Res Int 2019;2019:9853701.

38. Gandhi L, Rodríguez-Abreu D, Gadgeel S, et al. Pembrolizumab plus Chemotherapy in Metastatic NonSmall-Cell Lung Cancer. N Engl J Med 2018;378:2078-92.

39. Paz-Ares L, Luft A, Vicente D, et al. Pembrolizumab plus Chemotherapy for Squamous Non-Small-Cell Lung Cancer. N Engl J Med 2018;379:2040-51.

40. West H, McCleod M, Hussein M, et al. Atezolizumab in combination with carboplatin plus nab-paclitaxel chemotherapy compared with chemotherapy alone as firstline treatment for metastatic non-squamous non-smallcell lung cancer (IMpower130): a multicentre, randomised, open-label, phase 3 trial. Lancet Oncol 2019;20:924-37.

41. Socinski MA, Jotte RM, Cappuzzo F, et al. Atezolizumab for First-Line Treatment of Metastatic Nonsquamous NSCLC. N Engl J Med 2018;378:2288-301.

42. Paz-Ares L, Ciuleanu TE, Yu X, et al. LBA3 Nivolumab (NIVO) + platinum-doublet chemotherapy (chemo) vs chemo as first-line (1L) treatment (tx) for advanced nonsmall cell lung cancer (aNSCLC): CheckMate 227 - part 2 final analysis. Ann Oncol 2019;30:XI67-XI68.

43. Hellmann MD, Paz-Ares L, Bernabe Caro R, et al. Nivolumab plus Ipilimumab in Advanced Non-Small-Cell Lung Cancer. N Engl J Med 2019;381:2020-31.

44. Reck M, Ciuleanu TE, Dols MC, et al. Nivolumab (NIVO) + ipilimumab (IPI) + 2 cycles of platinum-doublet chemotherapy (chemo) vs 4 cycles chemo as first-line (1L) treatment (tx) for stage IV/recurrent non-small cell lung cancer (NSCLC): CheckMate 9LA. J Clin Oncol 2020;38:abstr 9501.

45. Horn L, Mansfield AS, Szczęsna A, et al. First-Line Atezolizumab plus Chemotherapy in ExtensiveStage Small-Cell Lung Cancer. N Engl J Med 2018;379:2220-9.

46. Paz-Ares L, Dvorkin M, Chen Y, et al. Durvalumab plus platinum-etoposide versus platinum-etoposide in firstline treatment of extensive-stage small-cell lung cancer (CASPIAN): a randomised, controlled, open-label, phase 3 trial. Lancet 2019;394:1929-39.

47. Ready NE, Ott PA, Hellmann MD, et al. Nivolumab Monotherapy and Nivolumab Plus Ipilimumab in Recurrent Small Cell Lung Cancer: Results From the CheckMate 032 Randomized Cohort. J Thorac Oncol 2020;15:426-35.

48. Wang DY, Salem JE, Cohen JV, et al. Fatal Toxic Effects Associated with Immune Checkpoint Inhibitors: A Systematic Review and Meta-analysis. JAMA Oncol 2018;4:1721-8.

49. Bilen MA, Martini DJ, Shabto JM, et al. Sarcopenia and inflammation predicts survival in advanced stage cancer patients (pts) treated with immunotherapy (IO). Ann Oncol 2018;29:VIII42.

50. Dall'Olio FG, Maggio I, Massucci M, et al. ECOG performance status $\geq 2$ as a prognostic factor in patients with advanced non small cell lung cancer treated with immune checkpoint inhibitors-A systematic review and meta-analysis of real world data. Lung Cancer 2020;145:95-104.

51. Iannuzzi-Sucich M, Prestwood KM, Kenny AM. Prevalence of sarcopenia and predictors of skeletal muscle mass in healthy, older men and women. J Gerontol A Biol Sci Med Sci 2002;57:M772-7.

52. Champiat S, Dercle L, Ammari S, et al. Hyperprogressive Disease Is a New Pattern of Progression in Cancer Patients Treated by Anti-PD-1/PD-L1. Clin Cancer Res 2017;23:1920-8.

53. Available online: https://www.ema.europa.eu/en/ documents/product-information/keytruda-epar-productinformation_en.pdf

54. Quoix E, Zalcman G, Oster JP, et al. Carboplatin and weekly paclitaxel doublet chemotherapy compared with monotherapy in elderly patients with advanced non-smallcell lung cancer: IFCT-0501 randomised, phase 3 trial. Lancet 2011;378:1079-88.

55. Available online: https://clinicaltrials.gov/ct2/show/NCT0 3293680 ? cond $=$ lung+cancer+elderly $\&$ draw $=3 \&$ rank $=17$

56. Available online: https://clinicaltrials.gov/ct2/show/NCT0 
3977194? cond=lung+cancer+elderly\&draw=3\&rank=10

57. Available online: https://clinicaltrials.gov/ct2/show/NCT0 4396457? cond=lung+cancer+elderly\&draw=3

58. Available online: https://clinicaltrials.gov/ct2/show/NCT0
4262869? term=elderly\&recrs $=$ ab\&cond $=$ Lung + Cancer + St age $+\mathrm{IV} \&$ draw $=2 \&$ rank $=3$

59. Available online: https://clinicaltrials.gov/ct2/show/ NCT03351361

Cite this article as: Naltet C, Besse B. Immune checkpoint inhibitors in elderly patients treated for a lung cancer: a narrative review. Transl Lung Cancer Res 2021;10(6):30143028. doi: 10.21037/tlcr-20-1239 